\title{
The educational impact of smartphone implementation in introductory mechanics laboratories
}

\author{
Colleen L. Countryman \\ North Carolina State University, Department of Physics, \\ Campus Box 8202, Raleigh, NC, 27606
}

\begin{abstract}
The internal sensors within students' smartphones are capable of collecting the data required of a traditional introductory mechanics laboratory curriculum. Some instructors have begun to implement these data collection devices into their labs. This report describes a project-entitled "MyTech," or "Measurements using everydaY TECHnologies"-that constitutes one such implementation. MyTech includes the development of a curriculum; the creation of a mobile app; and the determination of the impact of students' smartphones on their kinematics graphing skills and their attitudes regarding their laboratory. A battery of pre- and post-semester testing as well as video recordings allow for determination of these impacts. Administration of the CLASS, for example, indicates greater positive shifts in "real-world connections" for the section using smartphones. The project also involved development of a new tool for video analysis that aids in determining the nature of the student-equipment interactions during the labs.
\end{abstract}

PACS: $01.50 . \mathrm{Lc}, 01.50 . \mathrm{Pa}$

\section{INTRODUCTION}

In an increasingly "mobile-friendly" world, $84 \%$ of all college students in the U.S. own a smartphone [1]. Furthermore, $36 \%$ of these students say that they want to use mobile technologies in their classroom more often than they do now [1].

Smartphones contain internal accelerometers and gyroscopes that allow their users to collect data about the motion of the device through space. By taking advantage of these sensors, one can implement smartphones as data collection devices in introductory mechanics laboratories. This paper describes one such mechanics laboratory curriculum under the "MyTech" (Measurements using everydaY TECHnologies) project label.

Physics education researchers have had a long history of examining the impact of new technologies in the classroom. Priscilla Laws" "Workshop Physics" was among the first to include digitized data collection from various sensors [2]. Robert Beichner's VideoGraph software was an early effort that allowed students to directly connect video data of various objects in motion to graphs of their position and velocity through time [3]. More recently, smartphones' increasing popularity and extensive capabilities provide support for similar inclusion in the instructional laboratory.

One can argue that students' familiarity with their own personal devices could reduce the pedagogical barriers associated with data collection and potentially promote greater autonomy in students' experimental methods.

The existing mechanics laboratory curriculum at North Carolina State University was adapted to test the impact of using emerging smartphone technology on students. In addition to standard equipment such as carts, tracks, and stopwatches, the traditional mechanics laboratory curriculum utilizes motion detectors, photogates, and spring force sensors that connect to desktop computers via a digital interface with corresponding software. Instead of using the aforementioned sensors, students in the experimental MyTech section used only their smartphones' internal sensors and a webcam to collect all of the necessary data. The webcam was required to supplement the motion data from smartphone. While the internal accelerometer is quite responsive, the data that it produces is subject to drift [4]. Therefore, a webcam connected to a computer with video analysis software (such as Tracker [5]) could overcome this deficiency easily by providing students with the necessary velocity and/or position data. Students in the MyTech section were also given slightly less "cookbook"style guidance than the control sections to determine its impact on the nature of their laboratory experiences.

For example, in one lab on impulse and momentum, students are asked to verify the "momentum principle," or an alternate expression of Newton's Second Law,

$$
\vec{F}_{\square \square \square}=\frac{\Delta \vec{p}}{\Delta t},
$$

for a cart rolling down a track into a spring. In the traditional sections, students simultaneously collect data from spring force sensors and photogates to determine the force on the cart, $\vec{F}_{n e t}$, and the change in momentum of the cart, $\Delta \vec{p}$, immediately before and after collision with the spring. In the MyTech version of this lab, the smartphone is attached to the cart and data are collected simultaneously from the accelerometer and the webcam.

Students in the study made use of pre-existing free apps for both Android and iOS smartphones that allowed them to access the raw sensor data and email the file to themselves as a Comma-Separated Value (CSV) file, which could be opened and further analyzed in a spreadsheet program such 
as Microsoft Excel. Android owners used AndroSensor [6] and iPhone owners used SensorLog [7].

The project sought to determine the impact of smartphones on students in an instructional laboratory environment. Our study was constructed to answer the following two research questions:

1. How do smartphones impact students' kinematics graphing skills?

2. How do smartphones impact students' attitudes about their lab experience?

\section{METHODOLOGY}

We conducted this study with a subset of students in North Carolina State University's introductory calculusbased physics laboratories. These students are primarily engineering majors. We collected student data over the course of the Fall 2013, Spring 2014 and Fall 2014 semesters. Each semester contained a control section using the traditional equipment and an experimental section using smartphones. The students performed their labs in NC State's Qualitative Education Research Laboratory (QERL), which allows one to record student behavior with ceilingmounted cameras and tabletop microphones [8]. This room - and therefore each of these sections - was limited to 12 students and one graduate student teaching assistant. This provided us with $N=32$ control group students and $N=39$ MyTech students. The same teaching assistant led both sections in each semester of the study, however, we had a different TA each of the three semesters. TA consistency across semesters was difficult to obtain due to scheduling conflicts and graduate student availability.

Students in each of the sections were administered a series of pre- and post-semester tests, including the Test of Understanding Graphs - Kinematics (TUG-K) [9], the Colorado Learning Attitudes about Science Survey (CLASS) [10], the Computer Anxiety Rating Scale (CARS) [11] and a survey of their smartphone use outside of the classroom. (The E-CLASS [12], or the CLASS for Experimental Physics, would have been an ideal test for this study. However, the E-CLASS was first published in January 2013, and we were not aware of its existence until after the start of the study. For the sake of consistency, the CLASS was administered throughout the study with minimal changes, e.g., "classroom" changed to "laboratory.") Also, a smartphone app survey was administered during the third semester of the study to the students in the experimental section to help guide the development of a new MyTech app, designed with students' feedback in mind. Finally, videos of the last two semesters of QERL observation were also analyzed to determine temporal differences in student-equipment interactions between the two sections.

\section{A. Video Observation Tool}

The study generated a large quantity of videos in two semesters of two sections of four groups working through roughly ten laboratory activities (adding up to 157 videos, each over an hour in length). To analyze this body of data, we created a Video Observation Tool (VOT), inspired by Cassandra Paul's Real-Time Instructor Observation Tool [13]. The tool was generated using a Visual Basic for Applications script within Microsoft Excel. The tool allows a researcher to watch a video of students while simultaneously coding the video with customizable behavioral tags. (This script is still in the process of being fine-tuned for general use, and will be published elsewhere.) The tool produces temporal analyses of students' activities according to the researcher's colorcoded behavior tags. The VOT allows one to compile all of these temporal data and compare students' behaviors in the control group to those in the MyTech group.

This VOT protocol was verified using our qualitative observations of student-TA interactions that emerged from the analyzed VOT data. For example, the Spring 2014 TA was quieter and less proactive than the Fall 2014 TA. This manifested itself in students spending more time reading the manual and less time engaging the TA in discussions about concepts.

\section{RESULTS}

The brevity of this article dictates that we only discuss those results with the largest differences between the experimental and control sections. There were no statistically significant differences $(p \leq 0.05)$ in the kinematics graphing skills between the two sections. A larger sample size may reduce statistical uncertainty, but the scope of the present study did not allow for any such expansion.

While the overall CLASS results for the pre- to postsemester shifts in attitudes were similar for the control and MyTech groups, one striking difference emerged between the two sections in the "Real World Connections" category. The CLASS was designed to "measure various facets of student attitudes and beliefs about learning physics," and allows researchers to compare students' beliefs about physics to experts' beliefs [10]. As illustrated in fig. 1, students in the MyTech section demonstrate a slight shift in the positive direction along the horizontal axis, indicating fewer unfavorable attitudes $(+0.15)$ whereas students in the control section demonstrate a dramatic shift in the negative direction along this same axis, indicating increased unfavorable attitudes (-0.79). Shifts in items from the "Real World Connections" category of the CLASS are provided in table 1. We acknowledge that the app may not be the sole cause of these shifts. It is possible that the added agency of the new curriculum might also contribute to these changes. 


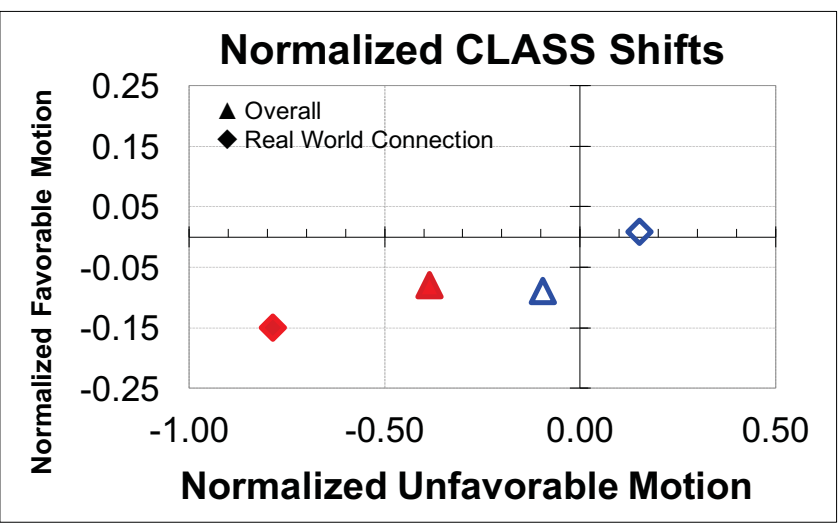

FIG 1. Normalized pre- and post-semester shifts in CLASS attitudinal results for the control group (red, solid) and the experimental MyTech group (blue, empty). "Favorable" motion is defined as a shift in students' beliefs toward experts' beliefs. A successful curriculum would result in all points being positioned in the first quadrant.

In table 1 , the bold typeface indicates the percentage of students that agree with experts' beliefs. The "novice" $(\mathrm{N})$ and "expert" (E) labels indicate low and high percentage shifts, respectively.

Video observations of students performing the labs in the QERL allowed access to a greater richness of data. Using the VOT, we were able to determine quantitatively the time spent on various activities in the lab. One of the more elucidating results emerged from the student-TA interactions.

As illustrated in fig. 2, the time that TAs spent aiding students with physical equipment (apparata devoid of electronics such as pendula, carts, tracks, rulers, and tape) was statistically significantly greater in the control sections than in the MyTech sections, despite the fact that none of the physical equipment varied between the two sections. The time that TAs spent helping students with misunderstanding an analytical tool (such as guiding them through Excel, or helping them with a difficult calculation) was larger in the MyTech sections than the control sections. Finally, students' difficulties with software malfunctions seemed to vary both with the section and the semester (i.e., TA) of the study.

TABLE 1. Shifts in items of the "Real World Connection" category in the CLASS.

\begin{tabular}{|c|c|c|c|c|c|c|}
\hline \multirow[b]{2}{*}{ Item } & \multicolumn{3}{|c|}{ MyTech } & \multicolumn{3}{|c|}{ Traditional } \\
\hline & Agree & $\begin{array}{c}\text { Dis- } \\
\text { agree }\end{array}$ & $\begin{array}{c}\text { Net } \\
\text { Shift }\end{array}$ & Agree & $\begin{array}{c}\text { Dis- } \\
\text { agree }\end{array}$ & $\begin{array}{l}\text { Net } \\
\text { Shift }\end{array}$ \\
\hline $\begin{array}{l}\text { "Learning physics } \\
\text { changes my ideas about } \\
\text { how the world works." }\end{array}$ & $4 \%$ & $-5 \%$ & E & $-1 \%$ & $4 \%$ & \\
\hline $\begin{array}{l}\text { "Reasoning skills used to } \\
\text { understand physics can } \\
\text { be helpful to me in my } \\
\text { everyday life." }\end{array}$ & $-1 \%$ & $3 \%$ & & $-4 \%$ & $3 \%$ & \\
\hline $\begin{array}{l}\text { "The subject of physics } \\
\text { has little relation to what I } \\
\text { experience in the real } \\
\text { world." }\end{array}$ & $1 \%$ & $-5 \%$ & & $7 \%$ & $-1 \%$ & \\
\hline $\begin{array}{l}\text { "To understand physics, I } \\
\text { sometimes think about } \\
\text { my personal experiences } \\
\text { and relate them to the } \\
\text { topic being analyzed." }\end{array}$ & $3 \%$ & $-5 \%$ & & $-12 \%$ & $15 \%$ & $\mathrm{~N}$ \\
\hline
\end{tabular}

\section{CONCLUSIONS AND DISCUSSION}

While the data from the pre- and post-semester CLASS indicate relatively little difference in the shifts of students' overall laboratory attitude, the items in the category of "Real World Connection" vary greatly between the two groups, as seen in fig. 1. These results lead us to consider the reasons for the differences in the CLASS results for the MyTech and control sections regarding the way in which students relate their physics laboratory experiences to their daily lives. First, perhaps, by MyTech students using their own personal devices, they are able to "insert themselves" into the physics experiment. For example, the MyTech lab requires students to consider the forces that their phone might experience as a bob on a swinging pendulum. This may strengthen their "Real World Connections." Secondly, one might posit that the mobile technology allows students to perform miniature physics experiments outside the laboratory, allowing them to further connect their physics concept knowledge to their daily lives.

Self-reports from technological usage surveys indicate that a few students voluntarily used the apps outside the classroom, such as while skateboarding down a hill or swinging their arms while walking.

Figure 2 indicates that time in the MyTech and control sections was utilized differently.

First, figure 2 illustrates that students in the control section requested more TA help with physical equipment.

Secondly, students in the MyTech section needed more help interpreting their analyzed data. We can attribute this to the slight differences in the MyTech lab manual. Students were required to consider some aspects of the data collection process in the MyTech section. For example, they had to determine the axis of interest on the accelerometer for each experiment. This additional cognitive load often led to some confusion and the necessity of additional time for analysis of the data.

Finally, an interesting pattern also arises from the time spent with the TA fixing a software malfunction. In Spring 2014, the TA spent more time helping students in the MyTech section, whereas the following semester, a different TA spent more time helping students in the control section. We believe that this disparity is largely due to the TAs' experience. The S14 TA had a great deal of experience troubleshooting issues in the prior control sections, and therefore did not require much time to fix issues in the control section of this study. However, the F14 TA had no prior experience with these labs. It stands to reason that the F14 TA required less time to troubleshoot the MyTech section (as compared to the control section) because we had predicted some common student issues with the MyTech laboratory manual. 


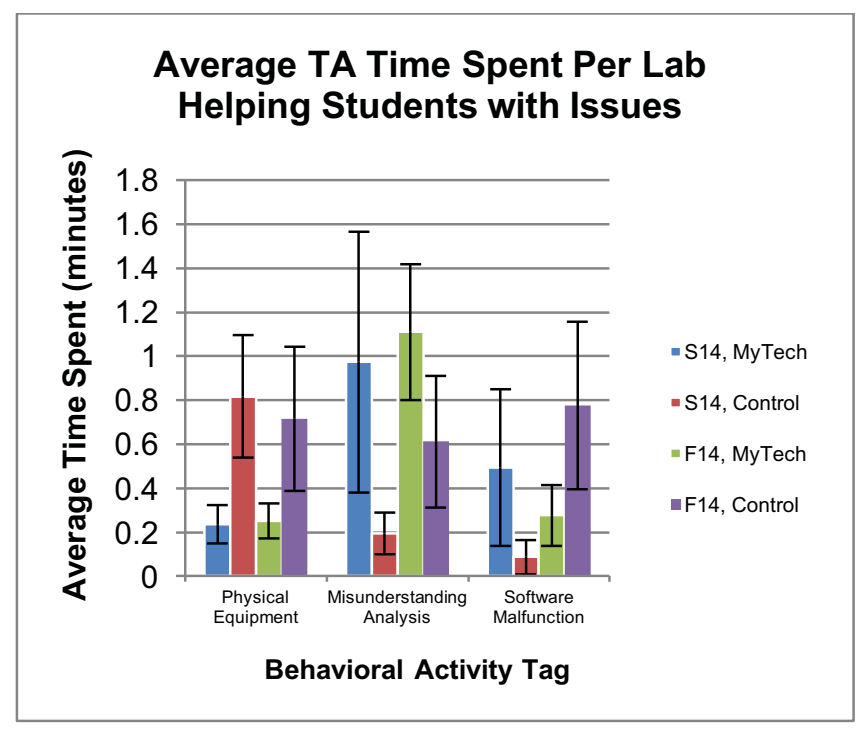

FIG 2. Teaching assistants spent statistically significant $(p$ $\leq 0.05$ ) more time helping students with physical equipment in the control sections than the MyTech sections. The time spent with TAs helping students work through software malfunctions, however, were less conclusive. Error bars indicate $95 \%$ confidence intervals.

Students' comments while using the MyTech equipment were mixed. Students indicated how pleasantly surprised they were at how well the equipment collected data ("It was pretty cool... that little graph. I was surprised by how smooth it was,") and how their data reinforced conceptual knowledge ("If I do this, I should get positive $z$... see? SCIENCE!"). However, students were also dismayed when the equipment did not work as they expected. Students and TAs alike often became frustrated when asked to interpret

[1] Harris Poll, Pearson Student Mobile Device Survey, http://www.pearsoned.com/wpcontent/uploads/Pearson-HE-Student-Mobile-DeviceSurvey-PUBLIC-Report-051614.pdf (2014).

[2] P. W. Laws, Calculus-based physics without lectures. Phys. Today, 44(12), 24-31 (1991).

[3] R. J. Beichner, Considering perception and cognition in the design of an instructional software package. Multimed. Tools Appl. 1(2), 173-184 (1995).

[4] G. Pang \& H. Liu, H, Evaluation of a low-cost MEMS accelerometer for distance measurement. J. Intell. Robot. Syst.: Theory Appl., 30, 249-265. (2001).

[5] D. Brown, Tracker Video Analysis and Modeling Tool for Physics Education. http://www.cabrillo.edu/ $\sim$ dbrown/tracker/ (2015).

[6] F. Asim. AndroSensor. https://play.google.com/store/ apps/details? $\mathrm{id}=$ com.fivasim.androsensor (2014).

[7] B. Thomas, SensorLog. https://itunes.apple.com/us/ app/sensorlog/id388014573?mt=8 (2011).

[8] STEM @ NCSU, Qualitative Education Research Lab, http://stem. ncsu.edu/ resources/qualitative.php the data with only some direct guidance from the instruction manual ("I think that's the $y$-axis...I am so confused.").

In order to improve the MyTech experience, we designed a MyTech app guided by students' feedback in the video observations and in an app survey administered during the last semester of the study.

Overall, the MyTech curriculum demonstrates promising attitudinal results, especially in the "Real World Connection" category and several important pedagogical implications resulted. Questions regarding the way in which the smartphone sensors collect data might only be more appropriate for advanced students. This is consistent with the most recent version of AAPT laboratory guidelines, which address specific differences in introductory and advanced students' interaction with laboratory equipment [14]. To administer a similar course, one should consider providing detailed instructions to guide introductory students' data collection methods. In addition, the high variability of time spent on various laboratory activities was largely dictated by the teaching assistant. A standardized and rigorous teaching assistant training program would increase the likelihood of success of a MyTech curriculum.

\section{ACKNOWLEDGEMENTS}

I would like to thank Dr. Robert Beichner, Dr. M. A. Paesler, William R. Sams and the entire Physics Education Research Group at NCSU from North Carolina State University. This project was made possible due to support from NSF (grant number 1245832, under the Directorate for Education and Human Resources) and North Carolina State University's DELTA Idea Grant Program.

[9] R. J. Beichner. Testing student interpretation of kinematics graphs. Am. J. Phys., 62(8), 750 (1994).

[10] W. K. Adams, The Design and Validation of the Colorado Learning Attitudes about Science Survey. AIP Conf. Proc., 790, 45-48 (2005).

[11] R. K. Heinssen, C. R. Glass, \& L. A. Knight, Assessing computer anxiety: Development and validation of the Computer Anxiety Rating Scale. Comput. Hum. Behav., 3(1), 49-59. (1987).

[12] B. M. Zwickl, N. Finkelstein, \& H. J. Lewandowski, The Process of Transforming an Advanced Lab Course: Goals, Curriculum, and Assessments. Am. J. Phys., 81(1), 63-70. (2013).

[13] E. A. West, C. A. Paul, C, D. Webb, \& W. H. Potter, Variation of instructor-student interactions in an introductory interactive physics course. Phys. Rev. Spec. Top. Ph., 9(1), 010109-1-010109-13. (2013).

[14] AAPT Committee on Laboratories. AAPT Recommendations for the Undergraduate Physics Laboratory Curriculum, 15. (2014) 